\title{
ON THE EQUATIONS $u_{t}+\nabla \cdot F(u)+0$ AND $u_{t}+\nabla \cdot F(u)=\nu \Delta u^{1}$
}

\author{
BY DANIEL B. KOTLOW
}

Communicated by Avner Friedman, June 6, 1969

This paper presents several results on global solutions of the initial value problems for the first order nonlinear conservation law

$$
u_{t}+\nabla \cdot F(u)=0
$$

and the associated second order nonlinear parabolic equation

$$
u_{t}+\nabla \cdot F(u)=\nu \Delta u, \quad \nu>0
$$

for an unknown scalar function $u=u(t, x)$ on the domain $D=$ $\left\{(t, x) \in R^{d+1} ; t>0\right\}$. Here $F \in C^{\infty}\left(R^{1}, R^{d}\right)$. For both equations, the given initial data are

$$
u(0, x)=u_{0}(x), \quad x \in R^{d} .
$$

We call these initial value problems $\operatorname{IVP}_{1}$ and $\mathrm{IVP}_{2}$ respectively. They are of interest as simplified prototypes of the initial value problems of gas dynamics (nonviscous and viscous respectively-cf. [2]).

We deal with weak solutions of $\operatorname{IVP}_{1}$ and $\operatorname{IVP}_{2}$. If $u \in L_{1}^{\text {loc }}(D)$, we say that $u$ is a weak solution of $I V P_{1}$ if for each $\phi \in C^{1}\left(R^{d+1}\right)$ of compact support

$$
\iint_{D}\left[u \phi_{t}+F(u) \cdot \nabla \phi\right] d x d t+\int_{R^{d}} u_{0}(x) \phi(0, x) d x=0 .
$$

We say that $u$ is a weak solution of $I V P_{2}$ if for each $\phi \in C^{2}\left(R^{d+1}\right)$ of compact support

$$
\iint_{D}\left[u \phi_{t}+\nu u \Delta \phi+F(u) \cdot \nabla \phi\right] d x d t+\int_{R^{d}} u_{0}(x) \phi(0, x) d x=0 .
$$

It is well known [2] that weak solutions of $\operatorname{IVP}_{1}$ are discontinuous and nonunique. For solutions of bounded variation locally in $D$, Vol'pert [3] has given a supplementary condition, called an entropy condition, on the discontinuities of a solution which singles out a unique solution in this class. We call this the entropy solution; it exists whenever $u_{0}$ is bounded and has bounded variation locally in $R^{d}[3]$.

${ }^{1}$ Publication was partially supported by National Science Foundation Grant NSFGP 5990 at Stanford University. 
We assume throughout that $u_{0}$ is integrable on $R^{d}$ and has bounded variation there. It follows that $\left|u_{0}\right| \leqq M$ for some $M$.

If $u(t, x)$ is a weak solution of $\mathrm{IVP}_{2}$ which is locally essentially bounded and has as distribution gradient $\nabla u(t, x)$ a bounded measure on compact sets of $D$, we call it a regular solution of $\mathrm{IVP}_{2}$.

THEOREM 1. If $u(t, x)$ is a regular solution of $I V P_{2}$, then $u \in C^{\infty}(D)$, equation (2) is satisfied in the classical sense in $D$, and the initial condition (3) is satisfied in the sense that, for each $\phi \in C^{0}\left(R^{d+1}\right)$ of compact support,

$$
\int_{R^{d}} u(t, x) \phi(t, x) d x \rightarrow \int_{R^{d}} u_{0}(x) \phi(0, x) d x \text { as } t \rightarrow 0 .
$$

THEOREM 2. IVP $P_{2}$ has at most one regular solution.

We approach the questions of existence and properties of solutions of $I V P_{1}$ and $I_{V P}$ through a finite difference scheme used by Conway and Smoller [1] to solve IVP. ${ }_{1}$. Our methods are slightly stronger than those of [1], and permit simultaneous consideration of $\operatorname{IVP}_{1}$ and $\mathrm{IVP}_{2}$.

Let $h, q>0$ be mesh lengths. Let $G$ be the $d$-dimensional lattice $G=\left\{x \in R^{d} ; x=q \alpha\right.$ for $\left.\alpha \in Z^{d}\right\}$. We label points in $G$ by their multiindices $\alpha$. Let $\delta(i)$ be the multi-index with 1 in the $i$ th component and 0 in all others. If $u$ is a function on $G, u^{\alpha}$ denotes its value at $\alpha \in G$. We consider maps $k \rightarrow u^{\alpha}(k)$ from the nonnegative integers to functions on $G$. Then our finite difference scheme may be written

$$
\begin{aligned}
h^{-1}\left[u^{\alpha}(k+1)-\right. & \left.(2 d)^{-1} \sum_{i=1}^{d}\left(u^{\alpha+\delta(i)}(k)+u^{\alpha-\delta(i)}(k)\right)\right] \\
& +\sum_{i=1}^{d}(2 q)^{-1}\left[F_{i}\left(u^{\alpha+\delta(i)}(k)\right)-F_{i}\left(u^{\alpha-\delta(i)}(k)\right)\right]=0
\end{aligned}
$$

with initial data

$$
u^{\alpha}(0)=u_{0}^{\alpha}
$$

where $F_{i}$ is the $i$ th component of $F$. It is clear that $u^{\alpha}(k)$ is uniquely determined through (6) by the initial data (7).

We may identify the $u^{\alpha}(k)$ (resp. $u_{0}^{\alpha}$ ) with functions $U(t, x)$ (resp. $U_{0}(x)$ ) which are constant on "grid cells." It is with this identification in mind that we speak of convergence of solutions of (6) (resp. convergence of the initial data (7)) as $h, q \rightarrow 0$. 
Let $h_{j}, q_{j} \rightarrow 0$ define a sequence of grids $G_{j}$. If $\left\|u_{0}^{\prime}\right\|_{\infty} \leqq M$, let $A=\max _{i}$ $\sup _{|v| \leq M}\left|F_{i}^{\prime}(v)\right|$, and assume that the stability condition $A d \leqq q_{j} / h_{j}$ holds for each $G_{j}$. It is possible to choose the initial data $U_{0}^{j}(x)$ so as to converge in $L_{1}\left(R^{d}\right)$ to $u_{0}$ with $\left\|U_{0}^{\prime}\right\|_{\infty} \leqq M$ and the total variation of $\nabla U_{0}^{j}$ bounded by that of $\nabla u_{0}$. We assume below that such a choice has been made.

Theorem 3. (i) If $q_{j}^{2} / 2 d h_{j} \rightarrow \nu>0$, then the finite difference solutions converge in $L_{1}^{\text {loc }}\left([0, T] \times R^{d}\right)$ for each fixed $T>0$ to a regular solution of $I V P_{2}$.

(ii) If $q_{j}^{2} / 2 d h_{j} \rightarrow 0$, then there is a subsequence of $G_{j}$ such that the finite difference solutions converge in $L_{1}^{\text {loc }}\left([0, T] \times R^{d}\right)$ for each fixed $T>0$ to a weak solution of IVP .

THEOREM 4. Let $u_{\nu}(t, x)$ be regular solutions of $I V P_{2}$ (parameterized by $\nu)$, and let $u(t, x)$ be the entropy solution of $I V P_{1}$. Then $u_{\nu}(t, x)$ $\rightarrow u(t, x)$ in $L_{1}^{\text {loc }}\left([0, T] \times R^{d}\right)$ as $\nu \rightarrow 0$ for each fixed $T>0$.

Some of these results were obtained in the author's doctoral dissertation at the Massachusetts Institute of Technology under the guidance of Professor James Glimm.

\section{REFERENCES}

1. E. Conway and J. Smoller, Global solutions of the Cauchy problem for quasilinear first order equations in several space variables, Comm. Pure Appl. Math. 19 (1966), 95105.

2. O. A. Oleinik, Discontinuous solutions of nonlinear equations, Uspehi Mat. Nauk 12 (1957), 3-73.

3. A. I. Vol'pert, The space $B V$ and quasilinear equations, Mat. Sb. 73 (1967), 255-302.

Massachusetts Institute of Technology, Cambridge, Massachusetts 02139 AND

Stanford University, Stanford, California 94305 